\title{
An improved postconditioning algorithm: Gradually increased reperfusion provides improved cardioprotection in rats
}

\author{
GUOMING ZHANG ${ }^{1,2^{*}}$, YUANYUAN SUN ${ }^{3 *}$, YU WANG $^{2}$, JING BAI $^{2}$, TIANDE LI $^{4}$, \\ XIAOYAN LI ${ }^{1}$, SHAOPING SU${ }^{5}$ and XIUHUA LIU ${ }^{6}$ \\ ${ }^{1}$ Department of Cardiology, General Hospital of Jinan Military Command, Jinan, Shandong 250031; ${ }^{2}$ Institute of Geriatric \\ Cardiology, Chinese PLA General Hospital, Beijing 100853; ${ }^{3}$ Department of Ultrasound, General Hospital of Jinan \\ Military Command, Jinan, Shandong 250031; ${ }^{4}$ Department of Cardiology, Cardiovascular Institute, ${ }^{5}$ Outpatient Department \\ of Geriatrics and ${ }^{6}$ Department of Pathophysiology, Chinese PLA General Hospital, Beijing 100853, P.R. China
}

Received January 12, 2013; Accepted June 10, 2013

DOI: $10.3892 / \mathrm{mmr} .2013 .1544$

\begin{abstract}
The aim of the present study was to investigate whether a gradually increasing reperfusion algorithm, in which the brief reperfusion was lengthened as the duration of each reperfusion/reocclusion cycle remained fixed, enhances cardioprotection. Rats were randomized into 5 groups: the sham, reperfusion injury (R/I), gradually decreased reperfusion (GDR; 30/10-25/15-15/25-10/30 sec of reperfusion/reocclusion), equal reperfusion (ER; 4 20/20-sec reperfusion/reocclusion cycles) and gradually increased reperfusion (GIR; 10/30-15/25-25/15-30/10 sec of reperfusion/reocclusion). The rats were sacrificed to measure serum markers, apoptotic indices and infarct size. Western blot analyses were used to analyze the expression of molecules involved in important signaling pathways. All the three postconditioning patterns were found to provide cardioprotection ( $\mathrm{P}<0.05$ compared with the $\mathrm{R} / \mathrm{I}$ group). GIR provided optimum cardioprotection, followed by ER and then GDR. Apoptotic index and serum marker levels were significantly reduced in the GIR compared with the ER group $(\mathrm{P}<0.05)$. The enhanced cardioprotection provided by GIR was accompanied by significantly increased levels of extracellular signal-regulated kinase 1/2 (ERK1/2) phosphorylation and Bcl-2, as well as lower levels of p38/c-Jun N-terminal kinase (JNK) phosphorylation, tumor necrosis factor $\alpha(\mathrm{TNF} \alpha)$, caspase- 8 , Bax, caspase- 9 and cytochrome $c(\mathrm{Cyt}-c)$ in the cytoplasm of rats $(\mathrm{P}<0.05$, all compared with ER). The infarct size in the rats of the GIR
\end{abstract}

Correspondence to: Dr Yu Wang or Dr Jing Bai, Institute of Geriatric Cardiology, Chinese PLA General Hospital, 28 Fuxing Road, Beijing 100853, P.R. China

E-mail: guomingpaper@126.com

E-mail: lifeinternet@126.com

*Contributed equally

Key words: ischemia-reperfusion injury, postconditioning, algorithm, apoptosis group was also smaller compared with that in the rats of the ER group, but this difference was not significant $(16.30 \pm 5.22$ vs. $20.57 \pm 6.32 \%, P>0.05)$. All the variables measured in the present study were significantly improved in the GIR group compared with the GDR group $(\mathrm{P}<0.05)$. In conclusion, the association between brief reperfusion and reocclusion is an important factor in postconditioning algorithms. Additionally, GIR results in improved cardioprotection compared with that achieved by the remaining algorithms examined.

\section{Introduction}

Rapid and complete reperfusion has been widely utilized in the treatment of patients with acute myocardial infarction. However, this process may lead to severe reperfusion injury (1-3). Since the study by Zhao et al (4) in 2003, mounting evidence has demonstrated that postconditioning potentially alleviates reperfusion injuries (5-8).

Postconditioning is performed according to a variety of protocols, known as postconditioning algorithms (9). However, no 'ideal' algorithm has been established as yet $(10,11)$. Various aspects of these algorithms have been investigated, such as the time interval from the end of ischemia to the application of postconditioning (12-14), the duration of reperfusion and reocclusion episodes $(15,16)$, and the number of cycles applied in the process (17). Postconditioning algorithms dictate when, how, and the duration required to apply reperfusion.

Limited data are available regarding the association between brief reperfusion and reocclusion in transient postconditioning. Penna et al (18) compared the effects of modified and classical postconditioning algorithms and found that the two algorithms yielded similar reductions in the infarct size and the release of lactate dehydrogenase. The total time differed between the two algorithms, with the classic algorithm lasting $10 \mathrm{sec}(5 \mathrm{cycles}$ of 10 -sec reperfusion/reocclusion) and the modified algorithm lasting $140 \mathrm{sec}(15 / 20-20 / 15-25 / 10-30 / 5 \mathrm{sec})$. In their study on postconditioning following brain ischemia, Wang et al (19) noted that when the brief reperfusion time was protracted and the reocclusion time had a short duration ( 3 cycles of $60 / 15 \mathrm{sec}$ of reperfusion/reocclusion), the protection was attenuated or even lost (19). 
In the present study, we hypothesized that protection is increased by gradually increasing the brief reperfusion time, decreasing the reocclusion time and keeping the total reperfusion/reocclusion cycle time fixed. This novel postconditioning algorithm is referred to as gradual increased reperfusion (GIR). Therefore, rats were subjected to acute myocardial infarctions, and the cardioprotection provided by GIR was compared with that resulting from standard postconditioning.

\section{Materials and methods}

Study approval. The present study conformed to the Guide for the Care and Use of Laboratory Animals published by the US National Institutes of Health (NIH publication no. 85-23, revised 1996) and was approved by the Research Commission on Ethics of the Chinese PLA General Hospital (Beijing, China).

Rat heart model of acute myocardial infarction and post-infarct treatment. Adult male Sprague-Dawley rats, weighing 200-250 g each, were fed a normal diet prior to the experiment. The rats were anesthetized with an initial intraperitoneal injection of sodium pentobarbital $(46 \mathrm{mg} / \mathrm{kg})$, and were then intubated and ventilated using a rodent respirator (ventilation rate, 52 breaths $/ \mathrm{min}$; tidal volume, $4.0 \mathrm{ml} / 100 \mathrm{~g}$ body weight). A parasternal incision was made to open the left pleural cavity by cutting the left four ribs and the intercostal muscle. Following pericardiotomy, a 5-0 ligature was placed under the left coronary artery by inserting the thread into the left atrium and threading it out from the side of the pulmonary artery cone. Prior to tying the knot, a balloon (Grip ${ }^{\mathrm{TM}}$, $3.0 \times 12 \mathrm{~mm}$; Acrostak, Geneva, Switzerland), connected to a pump full of water at a pressure of $1 \mathrm{~atm}$, was placed into the artery. After the knot was tied, the pressure of the balloon was immediately adjusted to $12 \mathrm{~atm}$ for $45 \mathrm{~min}$. After the $45-\mathrm{min}$ occlusion period, the pressure of the balloon was immediately adjusted to 0 for various periods of time, as per the protocols described below (Fig. 1). When the rats recovered from anesthesia, tracheal intubation was removed. The rats were anesthetized $24 \mathrm{~h}$ later with an initial intraperitoneal injection of urethane $(2 \mathrm{~g} / \mathrm{kg})$, and the right carotid artery was cannulated using an arterial catheter connected to a physiograph through a three-way stopcock. The hearts were then excised according to the following procedures: i) anterior wall tissue was obtained from the left ventricle after $6 \mathrm{~h}$ of reperfusion and maintained in a $-80^{\circ} \mathrm{C}$ freezer until western blot analysis; ii) the infarct size was measured after $24 \mathrm{~h}$ of reperfusion, and the hearts were immediately stained; iii) the myocardial tissue was soaked in formalin $(10 \%, \mathrm{pH} 7.4)$ until it was used for apoptotic index measurements.

Experimental protocols. Fifty rats were randomly allocated to one of the following 5 groups (Fig. 1): i) the sham group (control group), where the rats underwent thoracotomy without ischemic treatment; ii) the reperfusion-injury group $(\mathrm{R} / \mathrm{I})$, where the rats were administered routine ischemic-reperfusion treatment; iii) the gradually decreased reperfusion group (GDR), where the rats were administered 4 cycles of reperfusion and reocclusion at the onset of reperfusion with reperfusion/occlusion times of 30/10-25/15-15/25-10/30 sec
(160 sec total intervention time); iv) the equal reperfusion group (ER), where the rats were administered 4 cycles of 20/20 sec reperfusion/reocclusion beginning immediately at the onset of reperfusion (160 sec total intervention time); and v) the gradually increased reperfusion group (GIR), where the rats were administered 4 cycles of reperfusion/reocclusion at the onset of reperfusion with times of 10/30-15/25-25/15-30/10 sec (160 sec total intervention time).

Homodynamic measurements. The heart rate (HR) and arterial pressure were physiographically monitored through the arterial catheter. Subsequently, + mean delta pressure/delta time of the left ventricle $(+\mathrm{dP} / \mathrm{dt})$ and $-\mathrm{dP} / \mathrm{dt}$ were analyzed using the physiograph, and the rate-pressure product (RPP) was calculated as the product of the rate and the mean arterial pressure (MAP).

Measurement of infarct size. Infarct size was measured according to the method described by Kerendi et al (20). After $24 \mathrm{~h}$ of reperfusion, the ligature at the coronary occlusion site was permanently tied, and Evans blue solution (1\%, 3-5 ml) was injected into the aorta to demarcate the left ventricular area at risk (AAR). The heart was excised 10 min later and was maintained at $-20^{\circ} \mathrm{C}$ for $\sim 20 \mathrm{~min}$. The heart was then sliced into four sections ( $2-\mathrm{mm}$ thick) from the base to the apex for staining with triphenyltetrazolium chloride (TTC, 2\%) at $37^{\circ} \mathrm{C}$ to measure the area of necrosis (AN). The AN, AAR and the area of the left ventricle (LV) were determined by area analysis using Image-Pro Plus software (version 4.1; Media Cybernetics LP, Silver Spring, MD, USA). The infarct size was expressed as a percentage of the AAR (AN/AAR), and the ischemic area was calculated as AAR/LV.

Serum marker release. Serum levels of creatine kinase (CK) and the MB isoenzyme of creatine kinase (CK-MB) were analyzed using an automatic biochemistry analyzer.

Detection of apoptotic cells. Apoptotic cells were detected on transverse sections of the left ventricle using a DNA fragmentation detection kit (Roche Diagnostics GmbH, Mannheim, Germany) based on the terminal deoxynucleotidyl transferase-mediated dUTP nick end-labeling (TUNEL) method, according to the manufacturer's instructions. The results were quantified as the 'apoptotic index': the number of positively stained apoptotic cardiocytes/total number of cardiocytes counted x $100 \%$.

Western blot analysis. Western blot analysis was performed as previously described (21). Briefly, the left ventricular myocardium was homogenized in lysis buffer. After sodium dodecyl sulfate polyacrylamide gel electrophoresis (SDS-PAGE), the proteins were transferred to nitrocellulose membranes and incubated with antibodies against phospho-extracellular signalregulated kinase (p-ERK), phospho-p38 (p-p38), phospho-c-Jun N-terminal kinase (p-JNK), tumor necrosis factor $\alpha(\mathrm{TNF} \alpha)$, caspase-8, Bcl-2, Bax, caspase-9 and $\beta$-actin (all were mouse polyclonal antibodies diluted 1:200; Santa Cruz Biotechnology, Inc., Santa Cruz, CA, USA) for $6 \mathrm{~h}$ followed by incubation with a horseradish peroxidase (HRP)-conjugated secondary antibody. Antigen-antibody 


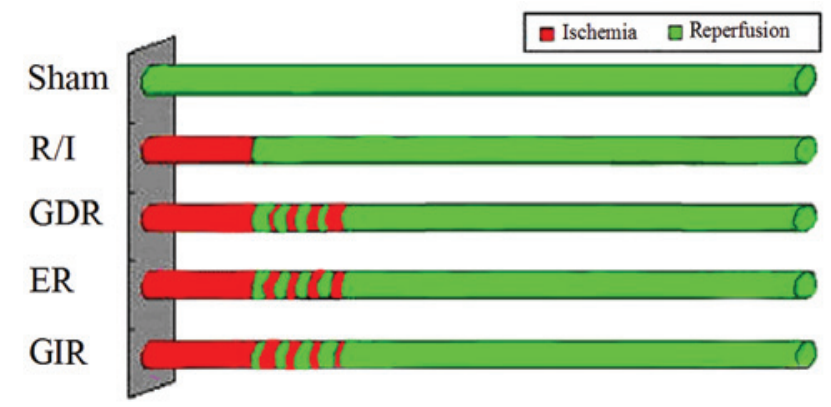

Figure 1. Experimental protocols demonstrating that GIR is more similar to gradual reperfusion compared to GDR and ER. R/I, reperfusion injury; GDR, gradually decreased reperfusion; ER, equal reperfusion; GIR, gradually increased reperfusion.

complexes were visualized using enhanced chemiluminescence (ECL).

Cytosolic and mitochondrial fractions were isolated as described by Li et al (21). The tissues were homogenized on ice using a tight-fitting Dounce homogenizer (Kimble Glass Inc., USA). The homogenates were centrifuged at 2,500 rpm for $10 \mathrm{~min}$ at $4^{\circ} \mathrm{C}$. The supernatants were then centrifuged at $14,000 \mathrm{rpm}$ for $25 \mathrm{~min}$ at $4^{\circ} \mathrm{C}$ to obtain the cytosolic fractions (supernatants) and mitochondrial fractions (pellets). Following SDS-PAGE, the proteins were transferred to nitrocellulose membranes and incubated with anti-cytochrome $c$ and $\beta$-actin antibodies (mouse polyclonal antibodies diluted 1:200; Santa Cruz Biotechnology, Inc.) for $6 \mathrm{~h}$ followed by incubation with an HRP-conjugated secondary antibody. Antigen-antibody complexes were visualized using ECL as described above.

Statistical analysis. Values were expressed as the mean \pm SEM. Data were analyzed using the statistical software package SPSS 13.0 for Windows. A one-way analysis of variance (ANOVA) was used, and the Student's t-test was employed to determine whether any significant differences in individual parameters existed between groups. $\mathrm{P}<0.05$ was considered to indicate a statistically significant difference.

\section{Results}

Hemodynamic data. Hemodynamic data are shown in Table I. Twenty-four hours after myocardial infarction, no significant differences in HR and MAP were observed between groups. The rats in the GIR group exhibited a significantly higher RPP value compared with the rats in the GDR and ER groups $(\mathrm{P}<0.05)$. Moreover, the $+\mathrm{dP} / \mathrm{dt}$ and $-\mathrm{dP} / \mathrm{dt}$ levels were significantly higher in the rats of the GIR group compared with the rats in the GDR group $(\mathrm{P}<0.05)$.

Serum markers of cardiac damage. The levels of CK and CK-MB release were significantly decreased in the three postconditioning groups $(\mathrm{P}<0.01$ compared with the $\mathrm{R} / \mathrm{I}$ group). The rats of the GIR group were found to release significantly less CK and CK-MB compared with the rats in the ER (CK, $251.00 \pm 45.16$ vs. $388.56 \pm 75.01 \mu / 1, \mathrm{P}<0.05$; CK-MB, $146.00 \pm 60.12$ vs. $291.16 \pm 52.41 \mu / 1, \mathrm{P} \leq 0.05)$ and GDR groups (CK, $251.00 \pm 45.16$ vs. 599.41 $\pm 63.00 \mu / 1, \mathrm{P}<0.05$; CK-MB, $146.00 \pm 60.12$ vs. $406.76 \pm 90.01 \mu / 1, \mathrm{P}<0.05)$.
A
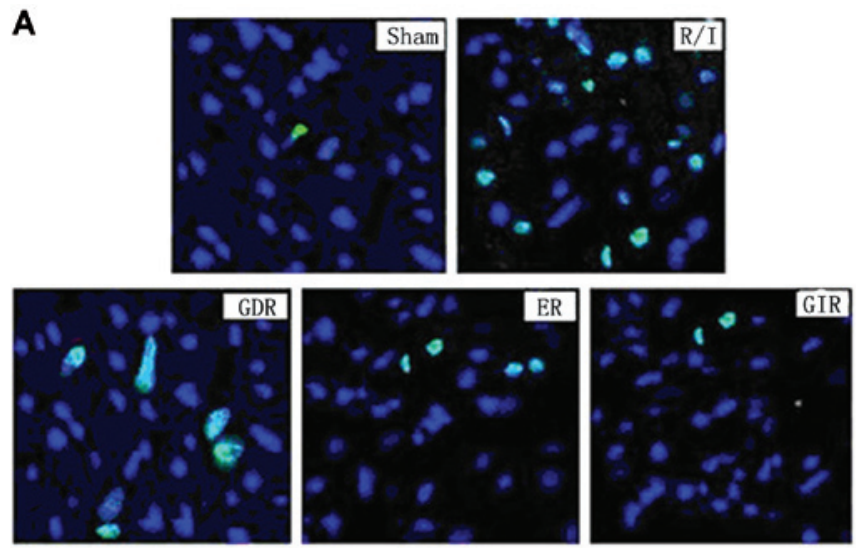

B

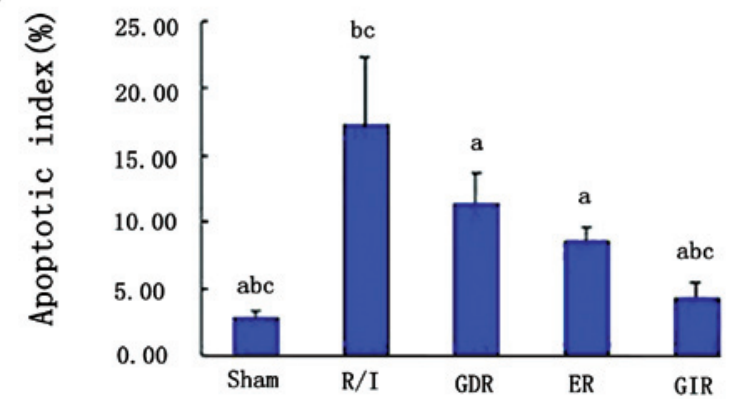

Figure 2. (A) Detection of apoptotic cells using TUNEL staining. TUNELpositive cells are indicated by green fluorescent dye. (B) Apoptotic index of all the groups. The apoptotic index of the GIR group was significantly lower compared with those in the R/I, GDR and ER groups $(\mathrm{P}<0.05)$. Values are the mean $\pm \mathrm{SEM}$. ${ }^{\mathrm{a}} \mathrm{P}<0.05$ vs. the $\mathrm{R} / \mathrm{I}$ group; ${ }^{\mathrm{b}} \mathrm{P}<0.05$ vs. the GDR group; ${ }^{\mathrm{c}} \mathrm{P}<0.05$ vs. the ER group. R/I, reperfusion injury; GDR, gradually decreased reperfusion; ER, equal reperfusion; GIR, gradually increased reperfusion.
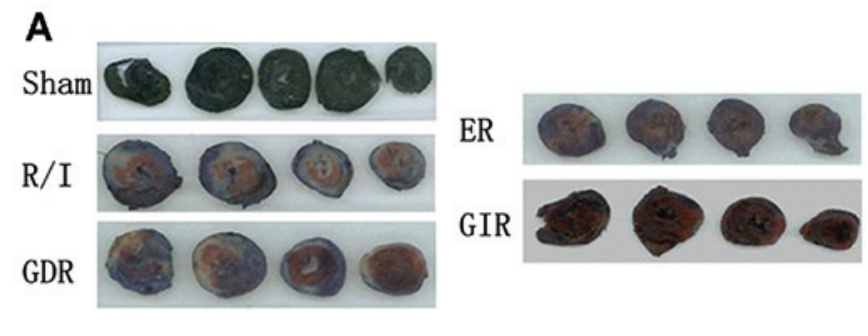

B

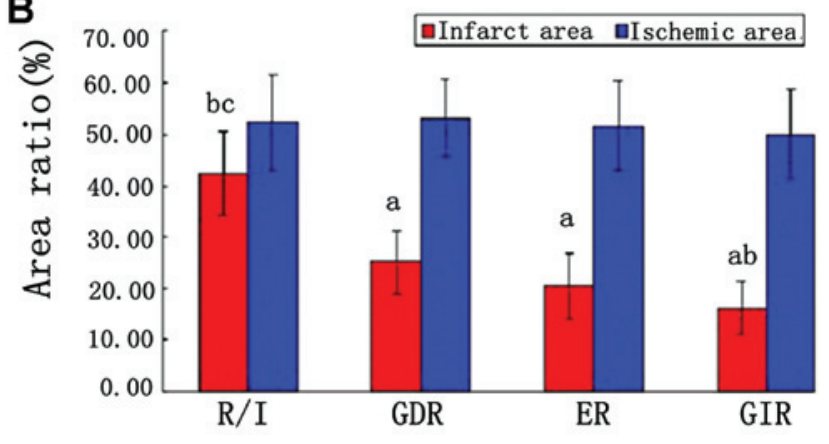

Figure 3. (A) Ischemic (AAR/LV) and infarct (AN/AAR) areas of all the groups. Blue indicates the non-ischemic zone (stained using Evans blue), red indicates ischemia but not infarct, and white indicates the infarct zone (stained using TTC). (B) The infarct size of the GIR group was significanlty smaller compared that of the R/I and GDR groups $(\mathrm{P}<0.05)$, while the difference between the GIR and ER groups was not significant $(\mathrm{P}>0.05)$. Values are the mean \pm SEM. ${ }^{a} \mathrm{P}<0.05$ vs. the $\mathrm{R} / \mathrm{I}$ group; ${ }^{\mathrm{b}} \mathrm{P}<0.05$ vs. the GDR group; ${ }^{\mathrm{c}} \mathrm{P}<0.05$ vs. the ER group. R/I, reperfusion injury; GDR, gradually decreased reperfusion; ER, equal reperfusion; GIR, gradually increased reperfusion. 
Table I. Hemodynamic data obtained $24 \mathrm{~h}$ following reperfusion $(\mathrm{n}=12)$.

\begin{tabular}{lccccc}
\hline Group & HR (beats/min) & MAP $(\mathrm{mmHg})$ & $\begin{array}{c}\text { RPP }(1,000 \mathrm{mmHg} \\
\text { beats } / \mathrm{min})\end{array}$ & $+\mathrm{dP} / \mathrm{dt}(\mathrm{mmHg} / \mathrm{sec})$ & $-\mathrm{dP} / \mathrm{dt}(\mathrm{mmHg} / \mathrm{sec})$ \\
\hline Sham & $418.00 \pm 65.93$ & $119.33 \pm 11.21$ & $49.35 \pm 0.74^{\mathrm{a}, \mathrm{b}}$ & $1,019.09 \pm 318.65^{\mathrm{a}, \mathrm{b}}$ & $752.31 \pm 231.05^{\mathrm{a}, \mathrm{b}}$ \\
R/I & $396.17 \pm 62.21$ & $105.95 \pm 31.68$ & $41.97 \pm 1.98^{\mathrm{b}, \mathrm{c}}$ & $526.37 \pm 123.99^{\mathrm{b}, \mathrm{c}}$ & $445.44 \pm 79.58^{\mathrm{b}, \mathrm{c}}$ \\
GDR & $423.21 \pm 50.00$ & $108.00 \pm 14.32$ & $45.70 \pm 0.72^{\mathrm{a}}$ & $721.45 \pm 89.97^{\mathrm{a}}$ & $546.23 \pm 100.12^{\mathrm{a}}$ \\
ER & $427.21 \pm 36.03$ & $109.57 \pm 10.36$ & $46.81 \pm 0.37^{\mathrm{a}}$ & $855.42 \pm 150.54^{\mathrm{a}}$ & $645.90 \pm 132.24^{\mathrm{a}}$ \\
GIR & $396.57 \pm 61.23$ & $123.00 \pm 15.42$ & $48.78 \pm 0.94^{\mathrm{a}-\mathrm{c}}$ & $913.24 \pm 63.25^{\mathrm{a}, \mathrm{b}}$ & $721.45 \pm 110.23^{\mathrm{a}, \mathrm{b}}$ \\
\hline
\end{tabular}

Data are the mean \pm SEM. ${ }^{\text {P }}<0.05$ vs. the I/R group; ${ }^{b} \mathrm{P}<0.05$ vs. the GDR group; ${ }^{c} \mathrm{P}<0.05$ vs. the ER group. HR, heart rate; MAP, mean arterial pressure; RPP, rate-pressure product/1000; dP/dt, mean delta pressure/delta time of the left ventricle; R/I, reperfusion injury; GDR, gradually decreased reperfusion; ER, equal reperfusion; GIR, gradually increased reperfusion.

Cardiocyte apoptosis. As shown in Fig. 2, R/I caused a significant increase in the number of TUNEL-positive cells in the rats of this group (apoptotic index, $\mathrm{P}<0.01$ ) compared with that of the rats in the sham group. All three postconditioning treatments significantly reduced the apoptotic index in the rats of the respective groups compared with that in the rats of the $\mathrm{R} / \mathrm{I}$ group $(\mathrm{P}<0.01)$. Furthermore, the apoptotic index in the GIR group was significantly lower compared with that in the ER $(4.32 \pm 1.16$ vs. $8.58 \pm 1.12 \%, \mathrm{P}<0.05)$ and GDR groups $(4.32 \pm 1.16$ vs. $11.34 \pm 2.34 \%, \mathrm{P}<0.05)$.

Infarct size. As shown in Fig. 3, the AAR zone is stained red and white, while the AN zone is stained white. The size of the ischemic area, expressed as a percentage of the area of the left ventricle (AAR/LV), was similar among the groups (51.01-53.55\%). Infarct size, expressed as a percentage of the area at risk (AN/AAR), was significantly reduced in all the postconditioning groups compared with the $\mathrm{R} / \mathrm{I}$ group $(\mathrm{P}<0.05)$. Furthermore, the infarct size of the GIR group was significantly smaller compared with that of the GDR $(16.30 \pm 5.22$ vs. $25.18 \pm 6.21 \%, \mathrm{P}<0.05)$ and $\mathrm{ER}$ groups $(16.30 \pm 5.22$ vs. $20.57 \pm 6.32 \%, \mathrm{P}<0.05)$, while the difference between the GIR and ER groups was not statistically significant.

Expression of the mitogen-activated protein kinases (MAPK) $p$-ERK1/2, p-p38 and p-JNK. The MAPK family is an important group of signaling molecules that includes ERK, which inhibits apoptosis and necrosis, and the apoptosis promoter p38/JNK. As shown in Fig. 4, a significant increase in the expression of $\mathrm{p}$-ERK and a marked decease in the expression of p-p38 and p-JNK were observed in all the postconditioning groups compared with the $\mathrm{R} / \mathrm{I}$ group $(\mathrm{P}<0.01)$. GIR treatment also enhanced the expression of $\mathrm{p}$-ERK to a greater extent compared with ER $(1.82 \pm 0.22$ vs. $1.54 \pm 0.32, \mathrm{P}<0.05)$ and GDR treatments $(1.82 \pm 0.22$ vs.1.22 $\pm 0.21, \mathrm{P}<0.01)$. The rats in the GIR group also exhibited a greater decrease in the levels of p-p38 and p-JNK compared with the rats in the ER (p-p38, $0.82 \pm 0.26$ vs. $1.63 \pm 0.24, \mathrm{P}<0.05$; p-JNK, $0.76 \pm 0.28$ vs. $1.33 \pm 0.21, P<0.05)$ and GDR groups $(\mathrm{p}-\mathrm{p} 38,0.82 \pm 0.26$ vs $1.98 \pm 0.21, \mathrm{P}<0.05 ; \mathrm{p}-\mathrm{JNK}, 0.76 \pm 0.28$ vs. $1.72 \pm 0.24, \mathrm{P}<0.05)$.

TNF $\alpha$ and caspase- 8 expression. TNF $\alpha$ and caspase- 8 are essential components of the death receptor apoptotic pathway;

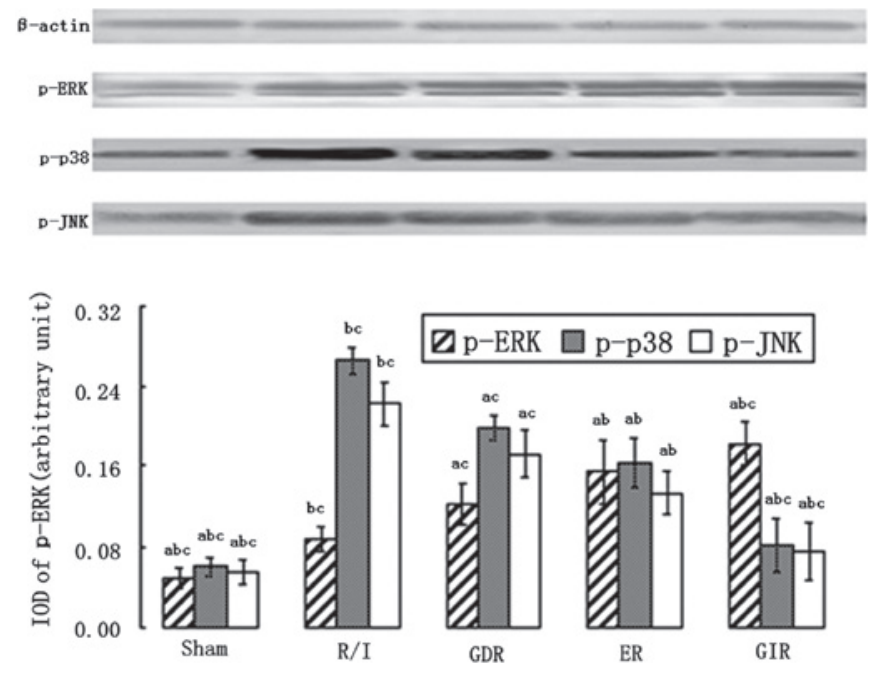

Figure 4. Expression of phosphorylated ERK (p-ERK), p38 (p-p38) and JNK (p-JNK) MAPK in all the groups. A significantly higher expression of p-ERK was observed in the GIR group. Lower levels of p-p38/JNK were also found in the GIR group compared with those in the R/I, GDR and ER groups $(\mathrm{P}<0.05)$. Values are the mean $\pm \mathrm{SEM}$. ${ }^{\mathrm{a}} \mathrm{P}<0.05$ vs. the $\mathrm{R} / \mathrm{I}$ group; ${ }^{\mathrm{b}} \mathrm{P}<0.05$ vs. the GDR group; ${ }^{c} \mathrm{P}<0.05$ vs. the ER group. R/I, reperfusion injury; GDR, gradually decreased reperfusion; ER, equal reperfusion; GIR, gradually increased reperfusion.

thus, their expression was measured in all the groups. As shown in Fig. 5, the rats in all the postconditioning groups exhibited a significantly lower expression of TNF $\alpha$ and caspase- 8 compared with the rats of the $\mathrm{R} / \mathrm{I}$ group $(\mathrm{P}<0.01)$. Furthermore, the TNF $\alpha$ and caspase- 8 expression levels in the GIR group were significantly lower compared with those in the ER (TNF $\alpha, 0.62 \pm 0.20$ vs. $1.00 \pm 0.12, \mathrm{P}<0.05$; caspase- 8 , $0.61 \pm 0.21$ vs. $1.00 \pm 0.21, \mathrm{P}<0.05)$ and GDR groups $(\mathrm{TNF} \alpha$, $0.62 \pm 0.20$ vs. $1.72 \pm 0.47, \mathrm{P}<0.05$; caspase- $8,0.86 \pm 0.21$ vs. $1.62 \pm 0.21, \mathrm{P}<0.05)$.

Bcl-2, Bax and caspase-9 expression. Bcl-2 and Bax are signaling molecules involved in the mitochondrial pathway of apoptosis, which is associated with the release of cytochrome $c$ (Cyt-c) from the mitochondrial matrix to the cytoplasm. As shown in Fig. 6, Bcl-2 was significantly upregulated in the rats of all the postconditioning groups compared with the rats of the $\mathrm{R} / \mathrm{I}$ group $(\mathrm{P}<0.01)$. The expression of $\mathrm{Bcl}-2$ in the GIR group 

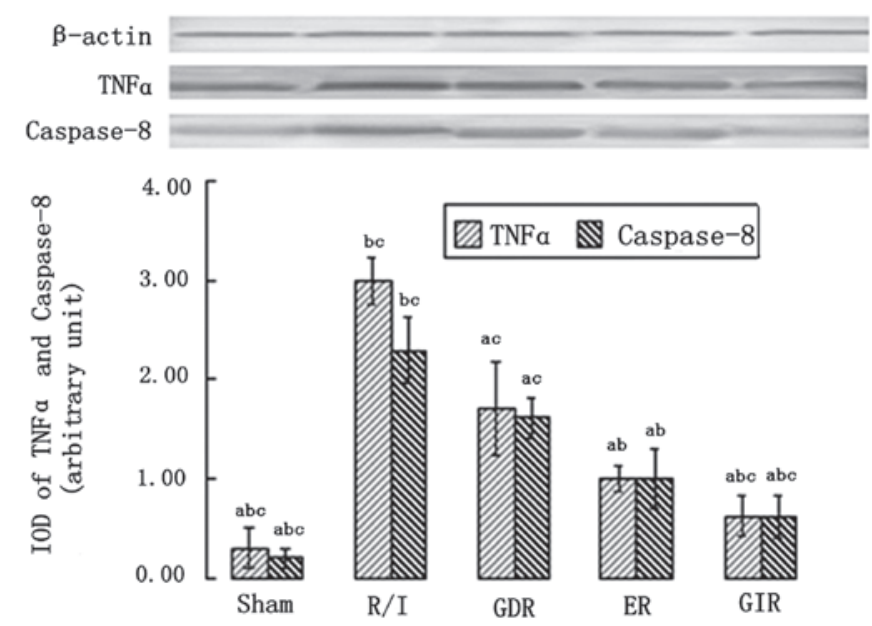

Figure 5. Expression of TNF $\alpha$ and caspase- 8 in all the groups. A lower expression of TNF $\alpha$ and caspase- 8 was found in the GIR group compared with the R/I, ER and GDR groups $(\mathrm{P}<0.05)$. Values are the mean \pm SEM. ${ }^{\mathrm{a}} \mathrm{P}<0.05$ vs. the $\mathrm{R} / \mathrm{I}$ group; ${ }^{\mathrm{b}} \mathrm{P}<0.05$ vs. the GDR group; ${ }^{\mathrm{P}} \mathrm{P}<0.05$ vs. the $\mathrm{ER}$ group. R/I, reperfusion injury; GDR, gradually decreased reperfusion; ER, equal reperfusion; GIR, gradually increased reperfusion.

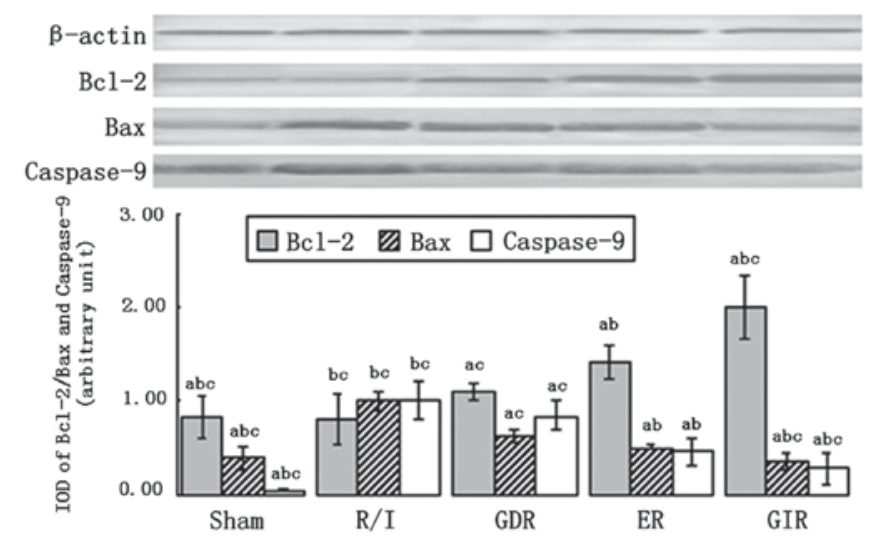

Figure 6. Expression of Bcl-2, Bax and caspase-9 in all the groups. A sig nificantly higher level of Bcl-2 was observed in the GIR group compared with the R/I, GDR and ER groups $(\mathrm{P}<0.05)$. However, lower levels of Bax and caspase- 9 were observed in the GIR group compared with the R/I, GDR and ER groups $(\mathrm{P}<0.05)$. Values are the mean $\pm \mathrm{SEM}$. ${ }^{\mathrm{a}} \mathrm{P}<0.05$ vs. the $\mathrm{R} / \mathrm{I}$ group; ${ }^{\mathrm{b}} \mathrm{P}<0.05$ vs. the GDR group; ${ }^{\mathrm{C}} \mathrm{P}<0.05$ vs. the ER group. $\mathrm{R} / \mathrm{I}$, reperfusion injury; GDR, gradually decreased reperfusion; ER, equal reperfusion; GIR, gradually increased reperfusion.

was significantly increased compared with that in the GDR $(2.00 \pm 0.34$ vs. $1.10 \pm 0.11, \mathrm{P}<0.05)$ and ER groups $(2.00 \pm 0.34$ vs. $1.40 \pm 0.18, \mathrm{P}<0.05)$. Moreover, the expression of Bax and caspase- 9 was decreased in all the postconditioning groups compared with the $\mathrm{R} / \mathrm{I}$ group $(\mathrm{P}<0.01)$. A significantly lower expression of Bax and caspase- 9 was observed in the GIR group compared with the GDR (Bax, $0.35 \pm 0.10$ vs. $0.62 \pm 0.07$, $\mathrm{P}<0.05$; caspase- $9,0.28 \pm 0.17$ vs. $0.84 \pm 0.16, \mathrm{P}<0.05)$ and $\mathrm{ER}$ groups ( $\mathrm{Bax}, 0.35 \pm 0.10$ vs. $0.50 \pm 0.02, \mathrm{P}<0.05$; caspase- 9 , $0.28 \pm 0.17$ vs. $0.46 \pm 0.15, \mathrm{P}<0.05)$.

Expression of Cyt-c in the cytosol. Cyt- $c$ is an important pro-apoptotic factor that activates caspase-9. As shown in Fig. 7, the rats of all the postconditioning groups exhibited a

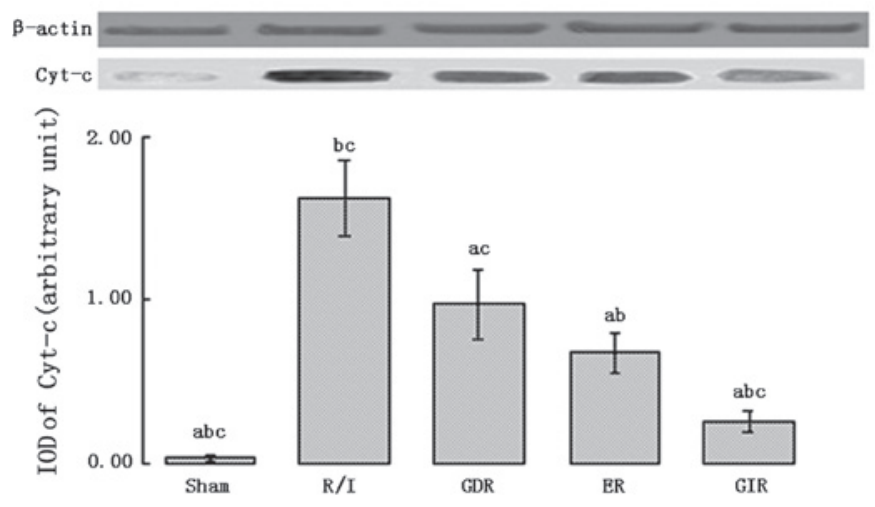

Figure 7. Cytosolic cytochrome $c(\mathrm{Cyt}-c)$ expression in all the groups. A significantly lower expression of cytosolic Cyt- $c$ was observed in the GIR group compared with the R/I, GDR and ER groups $(\mathrm{P}<0.05)$. Values are the mean \pm SEM. ${ }^{a} \mathrm{P}<0.05$ vs. the $\mathrm{R} / \mathrm{I}$ group; ${ }^{\mathrm{b}} \mathrm{P}<0.05$ vs. the GDR group; ${ }^{\mathrm{C}} \mathrm{P}<0.05$ vs. the ER group. R/I, reperfusion injury; GDR, gradually decreased reperfusion; ER, equal reperfusion; GIR, gradually increased reperfusion.

significantly lower Cyt- $c$ expression in the cytosol compared with the rats of the R/I group $(\mathrm{P}<0.01)$. Furthermore, the cytosolic Cyt $c$ level was lower in the GIR group compared with the ER $(0.26 \pm 0.06$ vs. $0.68 \pm 0.12, \mathrm{P}<0.05)$ and GDR groups $(0.26 \pm 0.06$ vs. $0.97 \pm 0.21, \mathrm{P}<0.05)$.

\section{Discussion}

The present study showed that GIR provides better cardioprotection compared with the remaining two postconditioning algorithms examined. Apoptosis and serum marker release were reduced to a greater extent in the GIR group compared with the ER group, while p-ERK and Bcl-2 were expressed at higher levels and TNF $\alpha$, caspase-8, Bax, caspase-9 and Cyt- $c$ were expressed at lower levels. GIR treatment also provided better protection compared with GDR treatment for all the variables measured in the present study.

The improved cardioprotection provided by GIR treatment compared with the remaining postconditioning algorithms investigated in the present study could be attributed to one or more of the following reasons. Firstly, results of previous studies have shown that ERK1/2 is one of the reperfusion injury survival kinases (RISK), which are components of an important postconditioning pathway $(14,22)$. In the present study, ERK1/2 was phosphorylated to higher levels in the GIR group compared with the ER and GDR groups. Myocardial ischemia/reperfusion has been demonstrated to activate p38/ JNK MAPK, resulting in cardiac injury and cell death, most prominently via apoptosis. According to a preliminary study on neonatal rat cardiocytes by Sun et al (23), hypoxic postconditioning was shown to inhibit the expression of $\mathrm{p} 38 / \mathrm{JNK}$ MAPK, reducing the expression of TNF $\alpha$ and Bax. Conversely, anisomycin, an activator of p38/JNK MAPK, was shown to offset this protection. In the present study, the level of phosphorylation of p38/JNK in the GIR group was lower compared with the R/I, ER and GDR groups, which was consistent with the results mentioned above.

Apoptosis is known to be an important component of reperfusion injury. The death receptor and mitochondrial pathways are the main pathways leading to cell apoptosis. The death 
receptor pathway is triggered by death receptors located on the cell membrane, such as TNF $\alpha$ receptor- 1 and the Fas receptor. In the present study, the expression of TNF $\alpha$ and caspase- 8 was significantly decreased in the GIR group compared with the ER group. The mitochondrial pathway is also important in apoptosis, and Bcl-2 and Bax are key factors that regulate this pathway (24-26). In the present study, the gradual increase in reperfusion time during postconditioning increased the expression of Bcl-2 and reduced the expression of Bax. These changes could lead to the opening of the mitochondrial permeability transition pore (mPTP), which is associated with apoptotic cell death since it controls the release of many pro-apoptotic factors, such as Cyt- $c$, in the cytoplasm (27-30). These pro-apoptotic factors could then activate many proenzymes, which could initiate the apoptotic cascade and trigger the release of molecules such as caspase- 9 and -3 .

The results of the present study indicated that there are some differences in the expression of downstream mediators among the GIR, ER and GDR postconditioning algorithms. Consequently, the underlying mechanisms of action of these algorithms that convert different mechanical manipulations into various biochemical signals should be subsequently investigated. Although this issue was not addressed in the present study, the gradual change of reperfusion time during postconditioning was demonstrated to be important. Postconditioning has been previously suggested to be a logical extension of gradual reperfusion, which is known to be cardioprotective for reperfusion injury (31-33). When a sudden change leads to an injury, a gradual change could attenuate the injury by alleviating the process. When myocardial cells remain viable following severe ischemia, a 'warm-up' period is required to reactivate their metabolic activity, and postconditioning leads to a gradual change in metabolic state. Consequently, the postconditioning process was modified accordingly to mimic gradual reperfusion. Among all the ischemia-postconditioning reperfusion algorithms examined in the present study, GIR was the postconditioning algorithm most similar to gradual reperfusion. The gradual change used in this algorithm resulted in improved cardioprotection compared with standard postconditioning, as described above.

In the present study, no significant difference in infarct size was observed between GIR and ER groups, possibly due to the small size. This finding could also be attributed to the fact that improvement in infarct size is difficult to be observed in the rat model. However, we could still conclude that GIR provides improved cardioprotection compared with ER treatment. Two main findings support this conclusion: i) GIR treatment led to a significant reduction in apoptosis and necrosis and markedly improved left ventricle function compared with ER treatment; and ii) GIR treatment improved all the variables examined compared with GDR treatment. These results clearly indicate that the gradual increase in the reperfusion time during postconditioning is associated with improved cardioprotection. Although the present study provides encouraging results, there are several limitations. These include the small sample size and the lack of comparison between GIR and gradual reperfusion.

In conclusion, the present study has demonstrated that the duration of the brief reperfusion and reocclusion steps in postconditioning algorithms is important, with the gradually increased reperfusion algorithm improving cardioprotection. However, future studies are needed to determine the underlying mechanisms of this phenomenon.

\section{Acknowledgements}

The authors are grateful to Sheng Sun, Lirong Cai, Feifei Xu, Xiaoreng Wang and Zhenying Zhang for their technical assistance. This study was supported by grants from the National Science Foundation of China (no. 30740080) and the Dean Fund of the General Hospital of Jinan Military Command (no. 2011Q08).

\section{References}

1. Wu JM, Wang ZR, Hsieh TC, Bruder JL, Zou JG and Huang YZ: Mechanism of cardioprotection by resveratrol, a phenolic antioxidant present in red wine (Review). Int J Mol Med 8: 3-17, 2001.

2. Braunwald E and Kloner RA: Myocardial reperfusion: a double-edged sword? J Clin Invest 76: 1713-1719, 1985.

3. Tomatsuri N, Yoshida N, Takagi T, et al: Edaravone, a newly developed radical scavenger, protects against ischemia-reperfusion injury of the small intestine in rats. Int J Mol Med 13: 105-109, 2004.

4. Zhao ZQ, Corvera JS, Halkos ME, et al: Inhibition of myocardial injury by ischemic postconditioning during reperfusion: comparison with ischemic preconditioning. Am J Physiol Heart Circ Physiol 285: H579-H588, 2003.

5. Sanada S, Komuro I and Kitakaze M: Pathophysiology of myocardial reperfusion injury: preconditioning, postconditioning, and translational aspects of protective measures. Am J Physiol Heart Circ Physiol 301: H1723-H1741, 2011.

6. Liu X, Chen H, Zhan B, et al: Attenuation of reperfusion injury by renal ischemic postconditioning: the role of NO. Biochem Biophys Res Commun 359: 628-634, 2007.

7. Zhang WH, Lu FH, Zhao YJ, et al: Post-conditioning protects rat cardiomyocytes via PKCepsilon-mediated calcium-sensing receptors. Biochem Biophys Res Commun 361: 659-664, 2007.

8. Thuny F, Lairez O, Roubille F, et al: Post-conditioning reduces infarct size and edema in patients with ST-segment elevation myocardial infarction. J Am Coll Cardiol 59: 2175-2181, 2012.

9. Penna C, Mancardi D, Raimondo S, Geuna S and Pagliaro P: The paradigm of postconditioning to protect the heart. J Cell Mol Med 12: 435-458, 2008

10. Skyschally A, van Caster P, Iliodromitis EK, Schulz R, Kremastinos DT and Heusch G: Ischemic postconditioning: experimental models and protocol algorithms. Basic Res Cardiol 104: 469-483, 2009.

11. Iliodromitis EK, Downey JM, Heusch G and Kremastinos DT: What is the optimal postconditioning algorithm? J Cardiovasc Pharmacol Ther 14: 269-273, 2009.

12. Cai M, Li Y, Xu Y, et al: Endothelial NOS activity and myocardial oxygen metabolism define the salvageable ischemic time window for ischemic postconditioning. Am J Physiol Heart Circ Physiol 300: H1069-H1077, 2011.

13. Kin H, Zhao ZQ, Sun HY, et al: Postconditioning attenuates myocardial ischemia-reperfusion injury by inhibiting events in the early minutes of reperfusion. Cardiovasc Res 62: 74-85, 2004.

14. Yang XM, Proctor JB, Cui L, Krieg T, Downey JM and Cohen MV: Multiple, brief coronary occlusions during early reperfusion protect rabbit hearts by targeting cell signaling pathways. J Am Coll Cardiol 44: 1103-1110, 2004.

15. Vinten-Johansen J, Zhao ZQ, Zatta AJ, Kin H, Halkos ME and Kerendi F: Postconditioning - A new link in nature's armor against myocardial ischemia-reperfusion injury. Basic Res Cardiol 100: 295-310, 2005.

16. Argaud L, Gateau-Roesch O, Raisky O, Loufouat J, Robert D and Ovize M: Postconditioning inhibits mitochondrial permeability transition. Circulation 111: 194-197, 2005.

17. Kin H, Zatta AJ, Lofye MT, et al: Postconditioning reduces infarct size via adenosine receptor activation by endogenous adenosine. Cardiovasc Res 67: 124-133, 2005. 
18. Penna C, Cappello S, Mancardi D, et al: Post-conditioning reduces infarct size in the isolated rat heart: role of coronary flow and pressure and the nitric oxide/cGMP pathway. Basic Res Cardiol 101: 168-179, 2006

19. Wang JY, Shen J, Gao Q, et al: Ischemic postconditioning protects against global cerebral ischemia/reperfusion-induced injury in rats. Stroke 39: 983-990, 2008.

20. Kerendi F, Kin H, Halkos ME, et al: Remote postconditioning. Brief renal ischemia and reperfusion applied before coronary artery reperfusion reduces myocardial infarct size via endogenous activation of adenosine receptors. Basic Res Cardio 100: 404-412, 2005

21. Li Y, Ge X and Liu X: The cardioprotective effect of postconditioning is mediated by ARC through inhibiting mitochondria apoptotic pathway. Apoptosis 14: 164-172, 2009.

22. Sumi S, Kobayashi H, Yasuda S, et al: Postconditioning effect of granulocyte colony-stimulating factor is mediated through activation of risk pathway and opening of the mitochondrial KATP channels. Am J Physiol Heart Circ Physiol 299: H1174-H1182, 2010.

23. Sun HY, Wang NP, Halkos M, et al: Postconditioning attenuates cardiomyocyte apoptosis via inhibition of JNK and p38 mitogen-activated protein kinase signaling pathways. Apoptosis 11: 1583-1593, 2006.

24. Gomez L, Thibault H, Gharib A, et al: Inhibition of mitochondrial permeability transition improves functional recovery and reduces mortality following acute myocardial infarction in mice. Am J Physiol Heart Circ Physiol 293: H1654-H1661, 2007.

25. Cao J, Zhu T, Lu L, et al: Estrogen induces cardioprotection in male C57BL/6J mice after acute myocardial infarction via decreased activity of matrix metalloproteinase- 9 and increased Akt-Bcl-2 anti-apoptotic signaling. Int J Mol Med 28: 231-237, 2011.
26. Mao H, Gu H, Qu X, et al: Involvement of the mitochondrial pathway and $\mathrm{Bim} / \mathrm{Bcl}-2$ balance in dihydroartemisinin-induced apoptosis in human breast cancer in vitro. Int J Mol Med 31: 213-218, 2013.

27. Hausenloy DJ, Ong SB and Yellon DM: The mitochondrial permeability transition pore as a target for preconditioning and postconditioning. Basic Res Cardiol 104: 189-202, 2009.

28. Paillard M, Gomez L, Augeul L, Loufouat J, Lesnefsky EJ and Ovize M: Postconditioning inhibits mPTP opening independent of oxidative phosphorylation and membrane potential. J Mol Cell Cardiol 46: 902-909, 2009.

29. Weiss JN, Korge P, Honda HM and Ping P: Role of the mitochondrial permeability transition in myocardial disease. Circ Res 93: 292-301, 2003.

30. Park SS, Zhao H, Mueller RA and Xu Z: Bradykinin prevents reperfusion injury by targeting mitochondrial permeability transition pore through glycogen synthase kinase 3beta. J Mol Cell Cardiol 40: 708-716, 2006

31. Sato H, Jordan JE,Zhao ZQ, Sarvotham SS and Vinten-Johansen J: Gradual reperfusion reduces infarct size and endothelial injury but augments neutrophil accumulation. Ann Thorac Surg 64: 1099-1107, 1997.

32. Unal S, Ozmen S, DemIr Y, et al: The effect of gradually increased blood flow on ischemia-reperfusion injury. Ann Plast Surg 47: 412-416, 2001.

33. Shi E, Jiang X, Kazui T, et al: Controlled low-pressure perfusion at the beginning of reperfusion attenuates neurologic injury after spinal cord ischemia. J Thorac Cardiovasc Surg 133: 942-948, 2007. 